\title{
Kernel-Based Interior-Point Methods for Monotone Linear Complementarity Problems over Symmetric Cones
}

\author{
G. Lesaja • C. Roos
}

Published online: 20 April 2011

(C) Springer Science+Business Media, LLC 2011

\begin{abstract}
We present an interior-point method for monotone linear complementarity problems over symmetric cones (SCLCP) that is based on barrier functions which are defined by a large class of univariate functions, called eligible kernel functions. This class is fairly general and includes the classical logarithmic function, the self-regular functions, as well as many non-self-regular functions as special cases. We provide a unified analysis of the method and give a general scheme on how to calculate the iteration bounds for the entire class. We also calculate the iteration bounds of both large-step and short-step versions of the method for ten frequently used eligible kernel functions. For some of them we match the best known iteration bound for large-step methods, while for short-step methods the best iteration bound is matched for all cases. The paper generalizes results of Lesaja and Roos (SIAM J. Optim. 20(6):3014$3039,2010)$ from $P_{*}(\kappa)$-LCP over the non-negative orthant to monotone LCPs over symmetric cones.
\end{abstract}

Keywords Linear complementarity problem - Euclidean Jordan algebras and symmetric cones · Interior-point method · Kernel functions · Polynomial complexity

Communicated by F.A. Potra.

G. Lesaja ( $\varangle)$

Department of Mathematical Sciences, Georgia Southern University, Statesboro, GA 30460-8093, USA

e-mail: goran@georgiasouthern.edu

C. Roos

Faculty of Electrical Engineering, Mathematics, and Computer Science, Delft University of Technology, P.O. Box 5031, 2600 GA Delft, The Netherlands 


\section{Introduction}

Let $(V, \circ)$ be an Euclidean Jordan Algebra (EJA) with rank $r$ equipped with the standard inner product $\langle x, y\rangle=\operatorname{tr}(x \circ y)$. Let $\mathcal{K}$ be the corresponding symmetric cone. The definitions of these and related concepts and results pertinent to the analysis presented in this paper are given in Sect. 2. We consider a class of monotone linear complementarity problems over symmetric cones (SCLCP):

Find $(x, s) \in \mathcal{K} \times \mathcal{K}$ such that

$$
(x, s) \in \mathcal{L}, \quad\langle x, s\rangle=0,
$$

where $\mathcal{L}$ is an affine subspace of finite dimension in $V \times V$. We assume that the affine subspace $\mathcal{L}$ has monotone property, that is, $\left\langle x_{1}-x_{2}, y_{1}-y_{2}\right\rangle \geq 0$ for each $\left(x_{1}, y_{1}\right)$ and $\left(x_{2}, y_{2}\right)$ in $\mathcal{L}$.

Although SCLCP is not an optimization problem, it is closely related to the optimization problems. One of the reasons is that optimality conditions of several important optimization problems can be written in the form of SCLCP. For example, the solution of a Linear Optimization (LO) problem over symmetric cones (SCLO) can be formulated as SCLCP.

The algebraic structure of EJA has been known for quite same time. However, surprisingly, in recent years it has been established that symmetric cones, which are cones of squares of EJAs, serve as a unifying framework to which the important cases of cones used in optimization, such as non-negative orthant, second order cone, and cone of positive semidefinite symmetric matrices (i.e., the positive semidefinite cone) belong. The classical monograph of Faraut and Koràny [2] provides a wealth of information on Jordan algebras, symmetric cones and related topics.

Nesterov and Nemirovski, in their classical monograph [3], were the first to study conic formulation of convex optimization problems. Later, Nesterov and Todd [4, 5], studied interior-point methods for convex optimization problems in the framework of self-scaled cones to which the non-negative orthant, second order cone, and positive semidefinite cone belong. However, they did not make the connection to EJA. Güler [6] was the first to observe that self-scaled cones are identical to symmetric cones for which there exists a complete classification in the setting of EJA.

Faybusovich was the first to analyze a short-step path-following IPM for SCLO and SCLCP [7, 8]. Subsequently, primal-dual IPMs for SCLO were analyzed by Alizadeh and Schmieta [9, 10]. In their Ph.D. theses (directed by the second author of this paper) Vieira [11] analyzed kernel-based IPMs for SCLO and Gu [12] analyzed full-Newton step IPMs for SCLO. While there is an extensive literature on IPMs for LCPs over the non-negative orthant (see for example [1, 13-21], to mention just a few), there are fewer results on LCPs over the cone of semidefinite matrices (see for example [22-24]), and even less so for LCP over general symmetric cones. In addition to Faybusovich's results, Rangarajan [25] proposed the first infeasible IPM for SCLCP. Yoshise $[26,27]$ was the first to analyze IPMs for Nonlinear Complementarity Problems (NCP) over symmetric cones.

All of the above mentioned results were obtained by using the classical logarithmic barrier function adapted for the framework of symmetric cones. The only result for IPMs with other types of barrier (kernel) functions, the eligible kernel functions, was obtained by Vieira [11], and only for SCLO. In this paper we consider 
a generalization of kernel-based IPMs to SCLCP. The obtained complexity results match the best known iteration bounds known for the large-update methods, namely $O\left(\sqrt{r} \log r \log \frac{r}{\epsilon}\right)$.

The outline of the paper is as follows. In Sect. 2, we outline some basic facts on EJA and the corresponding symmetric cones, which will be needed in subsequent sections of the paper with the intent to make the paper more self-contained and easy to follow. In Sect. 3, we give a Generic IPM to solve monotone SCLCP that is based on the use of a general barrier function. In Sect. 4, we consider a wide class of socalled eligible kernel functions and barrier functions based on them, and discuss some of the properties in particular as they relate to symmetric cones. This class of kernel functions was first introduced by Bai et al. in [28]. In Sect. 5, we provide an analysis of the Generic IPM outlined in Sect. 3 based on the barrier functions defined by eligible kernel functions. In Sect. 6, the complexity of the method is discussed. In Sect. 7, a unified scheme for the calculation of iteration bounds is given and it is applied to a number of specific eligible kernel functions. Concluding remarks are presented in Sect. 8.

\section{Preliminaries}

In order to make the paper self-contained and easy to follow, in this section we briefly review main definitions, concepts, and results on EJA and symmetric cones that are used, directly or indirectly, in the subsequent sections of the paper. A more detailed review can be found in $[2,11,12,29]$.

Definition 2.1 A pair $(V, \circ)$ is called Jordan algebra iff $V$ is a finite-dimensional vector space over $\mathbf{R}$ and $\circ: V \times V \rightarrow V$ is bilinear form (product) with properties

(i) $x \circ y=y \circ x$,

(ii) $x \circ\left(x^{2} \circ y\right)=x^{2} \circ(x \circ y)$, (Jordan Axiom).

A Jordan algebra is not necessarily associative; however, it is power associative, that is, $x^{p} \circ x^{q}=x^{p+q}$ (Proposition II.1.2 in [2]). In the sequel, we will also assume that a Jordan algebra is equipped with an identity element $e$, i.e., $x \circ e=e \circ x=x$ for each $x \in V$.

Let $\mathbf{R}[X]$ denote the algebra over $\mathbf{R}$ of polynomials in one variable $X$ with coefficients in $\mathbf{R}$. For $x \in V$ we define

$$
\mathbf{R}[x]:=\{p(x): p \in \mathbf{R}[X]\} .
$$

Since $V$ is a finite-dimensional vector space, for each $x \in V$ there exists a positive integer $k \leq \operatorname{dim}(V)$, such that $e, x, x^{2}, \ldots, x^{k}$ are linearly dependent. This implies the existence of a non-zero polynomial $p \in \mathbf{R}[X]$, such that $p(x)=0$. Among all such polynomials the one that has a leading coefficient equal to 1 (monic polynomial) and is of minimal degree is called the minimal polynomial of $x \in V$. It is not hard to show that the minimal polynomial of $x \in V$ is unique.

The degree of $x \in V$ is defined as the degree of the associated minimal polynomial of $x$. It is denoted as $\operatorname{deg} x$. Obviously, $\operatorname{deg} x \leq \operatorname{dim} V$, where $\operatorname{dim} V$ denotes the 
dimension of $V$. The maximal degree over all elements in $V$ is called the rank of $V$, i.e.

$$
r:=\operatorname{rank} V:=\max \{\operatorname{deg} x: x \in V\} .
$$

Element $x \in V$ is called regular iff $\operatorname{deg} x=r$.

An important result regarding minimal polynomials of regular elements is the following lemma.

Lemma 2.1 (Proposition II.2.1 in [2]) The set of regular elements is open and dense in $V$. There exist polynomials $a_{1}, a_{2}, \ldots, a_{r} \in \mathbf{R}[X], i=1, \ldots, r$ such that $a_{i}(x) \in \mathbf{R}$ and the minimal polynomial of every regular element $x \in V$ in the variable $\lambda$ is given by

$$
f(\lambda ; x)=\lambda^{r}-a_{1}(x) \lambda^{r-1}+a_{2}(x) \lambda^{r-2}+\cdots+(-1)^{r} a_{r}(x) .
$$

The polynomials $a_{1}, \ldots, a_{r}$ are unique and $a_{j}$ is homogeneous of degree $j$, for $j=$ $1, \ldots, r$.

The polynomial $f(\lambda ; x)$ is called the characteristic polynomial of a regular element $x \in V$. Since regular elements are dense in $V$, the characteristic polynomial can be defined for all elements in $V$. Furthermore, the coefficients $a_{1}(x)$ and $a_{r}(x)$ have special names; they are called trace and determinant and have the following notation:

$$
\operatorname{tr}(x)=a_{1}(x), \quad \operatorname{det}(x)=a_{r}(x) .
$$

The definition of $\mathbf{R}[x]$ was instrumental in defining the minimal and characteristic polynomial of $x \in V$. This definition also allows us to define the inverse element of $x \in V$ if it exists. We say that $y \in \mathbf{R}[x]$ is the inverse of $x$ iff $x \circ y=e$. Since $\mathbf{R}[x]$ is associative, $y$ is unique and it is denoted as $x^{-1}$.

The concepts of Multiplication and Quadratic Representation Operators of element $x \in V$ are essential in the analysis of Jordan algebras and related topics. They also play a central role in proving the results in this paper.

The Multiplication Operator of $x \in V$ is the linear operator $L: V \rightarrow V$ such that

$$
L(x) y:=x \circ y, \quad \forall y \in V .
$$

$L(x)$ is linear in $x$ and $L(e)$ is the identity operator, which we also denote by $I$. Using the above definition, the axioms in Definition 2.1 of Jordan algebra are equivalent to

(i*) $L(x) y=L(y) x, \forall x, y \in V$,

(ii*) $L(x) L\left(x^{2}\right)=L\left(x^{2}\right) L(x), \forall x \in V$, (Jordan Axiom).

In this context, the Jordan Axiom means that the operators $L(x)$ and $L\left(x^{2}\right)$ commute.

It turns out that even more useful is the Quadratic Representation Operator defined as

$$
P(x):=2 L(x)^{2}-L\left(x^{2}\right) .
$$

Operators $L(x)$ and $P(x)$ are related to the inverse element of $x \in V$ in the following way. If $L(x)$ is invertible, then $x$ is invertible and $x^{-1}=L(x)^{-1} e$. Unfortunately, 
the converse is not always true (Proposition II.2.3 in [2]). However, $P(x)$ is invertible, if and only if $x$ is invertible (Proposition II.3.1 in [2]). In this case

$$
\begin{gathered}
P(x) x^{-1}=x, \\
P(x)^{-1}=P\left(x^{-1}\right), \\
(P(x) y)^{-1}=P\left(x^{-1}\right) y^{-1} .
\end{gathered}
$$

In addition, the following important formula, called the Fundamental Formula, holds (Proposition II.3.3 in [2]):

$$
P(P(y) x)=P(y) P(x) P(y) .
$$

The fundamental formula has many consequences, one of which is often used (Corollary 2.3.9 in [11]),

$$
P\left(x^{k}\right)=P(x)^{k} .
$$

There are numerous additional interesting properties of these operators not mentioned here. For more information the interested reader is referred to [2, 11, 12].

In this paper, we are especially interested in Euclidean Jordan algebras (EJAs). A Jordan algebra is called Euclidean iff it is equipped with a positive definite symmetric bilinear form, or equivalently, the inner product $\langle x, y\rangle$ that is associative

$$
\langle x \circ z, y\rangle=\langle x, z \circ y\rangle, \quad \forall x, y, z \in V .
$$

It can be proved that $\operatorname{tr}(x \circ y)$ satisfies the properties of inner product (Proposition II.4.3 and Theorem III.1.5 in [2]). Thus, we can define

$$
\langle x, y\rangle:=\operatorname{tr}(x \circ y)
$$

In an EJA, every element has a Spectral decomposition which uses the notion of a Jordan frame. First, an element $c \in V$ is called an idempotent element iff $c^{2}=c$. We say that an idempotent is primitive iff it is non-zero and cannot be written as the sum of two (necessarily orthogonal) non-zero idempotents. A set $\left\{c_{1}, \ldots, c_{m}\right\}$ is a complete system of orthogonal primitive idempotents, or a Jordan frame, if each $c_{j}$ is a primitive idempotent and if

$$
\begin{aligned}
& c_{i} \circ c_{j}=0, \quad i \neq j, \\
& \sum_{j=1}^{m} c_{j}=e .
\end{aligned}
$$

The importance of Jordan frame comes from the fact that any element of EJA can be represented using some Jordan frame, as explained more precisely in the following theorem. 
Theorem 2.1 (Theorem III.1.2 in [2]) Suppose $V$ is EJA with rank $r$. Then for every $x \in V$ there exists a Jordan frame $c_{1}, \ldots, c_{r}$ and real numbers $\lambda_{1}(x), \ldots, \lambda_{r}(x)$ such that

$$
x=\sum_{j=1}^{r} \lambda_{j}(x) c_{j}
$$

The numbers $\lambda_{j}(x)$ (with their multiplicities) are uniquely determined by $x$ and are called eigenvalues of $x$. Furthermore,

$$
\operatorname{det}(x)=\prod_{j=1}^{r} \lambda_{j}(x), \quad \operatorname{tr}(x)=\sum_{j=1}^{r} \lambda_{j}(x) .
$$

More generally,

$$
a_{k}(x)=\sum_{1 \leq i_{1} \leq \cdots \leq i_{k} \leq r} \lambda_{i_{1}}(x) \cdots \lambda_{i_{k}}(x),
$$

where $a_{k}(1 \leq k \leq r)$ are the polynomials that are coefficients of the characteristic polynomial $f(x ; \lambda)$.

An important consequence of the above theorem is that the eigenvalues of $x$ are real numbers and they are exactly the zeros of the characteristic polynomial. Furthermore, given the definition of the Jordan frame, it immediately follows that the unit element $e$ has only 1 as its eigenvalue with multiplicity $r$.

The norm induced by the inner product (5) is called the Frobenius norm

$$
\|x\|_{F}:=\sqrt{\langle x, x\rangle} .
$$

Using the spectral decomposition of $x$, that is, $x=\sum_{i=1}^{r} \lambda_{i}(x) c_{i}$, which implies $x^{2}=$ $\sum_{i=1}^{r} \lambda_{i}^{2}(x) c_{i}$, the norm can be expressed as follows:

$$
\|x\|_{F}=\sqrt{\langle x, x\rangle}=\sqrt{\operatorname{tr}\left(x^{2}\right)}=\sqrt{\sum_{i=1}^{r} \lambda_{i}^{2}(x)}=\|\lambda(x)\|,
$$

where $\lambda(x)$ is a vector of eigenvalues of $x$. Often, when there is no danger of confusion, the subscript in the definition of the Frobenius norm will be omitted.

Useful eigenvalue inequalities, which will be needed in subsequent sections, are given in the following lemma.

Lemma 2.2 (Lemma 14 in [10]) Let $a, b \in V$; then we can bound the eigenvalues of $a+b$ as follows:

$$
\begin{aligned}
& \lambda_{\min }(a+b) \geq \lambda_{\min }(a)-\|b\|, \\
& \lambda_{\max }(a+b) \leq \lambda_{\max }(a)+\|b\| .
\end{aligned}
$$


There are other important eigenvalue inequalities that have been used indirectly in the proofs of some results stated in this section. They can be found in [11], Chap. 3.

Another important decomposition of an element $x \in V$ used in the paper is the Peirce decomposition. It is based on the fact that, for the idempotent $c \in V$, the only eigenvalues of $L(c)$ are $0,1 / 2$, and 1 (Proposition III.1.3 in [2]). Furthermore, the eigenspaces corresponding to these eigenvalues are

$$
V(c, \lambda):=\{x \in V: L(x) c=c \circ x=\lambda x\}, \quad \lambda \in\left\{0, \frac{1}{2}, 1\right\} .
$$

Hence, $V$ is the direct sum of the corresponding subspaces $V(c, 1), V\left(c, \frac{1}{2}\right)$ and $V(c, 0)$. The decomposition

$$
V=V(c, 1) \oplus V\left(c, \frac{1}{2}\right) \oplus V(c, 0)
$$

is called the Peirce decomposition of $V$ with respect to the idempotent $c$. It is not hard to show that the decomposition is orthogonal with respect to the inner product in $V$. Consequently, one can define a Pierce decomposition with respect to a Jordan frame.

Theorem 2.2 (Theorem IV.2.1 in [2]) Let $V$ be an Euclidean Jordan algebra and $\left\{c_{1}, \ldots, c_{r}\right\}$ a Jordan frame. Then

(i) V can be decomposed into the direct sum

$$
V=\bigoplus_{i \leq j} V_{i j}
$$

where

$$
\begin{aligned}
V_{i i} & :=V\left(c_{i}, 1\right)=\mathbf{R} c_{i}, \\
V_{i j} & :=V\left(c_{i}, \frac{1}{2}\right) \cap V\left(c_{j}, \frac{1}{2}\right), \quad i \neq j .
\end{aligned}
$$

(ii) If we denote by $P_{i j}$ the orthogonal projection onto $V_{i j}$, then

$$
\begin{aligned}
& P_{i i}=P\left(c_{i}\right), \\
& P_{i j}=4 L\left(c_{i}\right) L\left(c_{j}\right), \quad i \neq j .
\end{aligned}
$$

(iii) Furthermore,

$$
\begin{aligned}
& V_{i j} \circ V_{i j} \subset V_{i i}+V_{j j}, \\
& V_{i j} \circ V_{j k} \subset V_{i k}, \quad \text { if } i \neq k, \\
& V_{i j} \circ V_{k l}=\{0\}, \quad \text { if }\{i, j\} \cap\{k, l\}=\emptyset .
\end{aligned}
$$


As a consequence the Peirce decomposition of $x \in V$ with respect to the Jordan frame $c_{1}, \ldots, c_{r}$ is given by

$$
x=\sum_{i=1}^{r} x_{i} c_{i}+\sum_{i<j} x_{i j}
$$

with $x_{i} \in \mathbf{R}, i=1, \ldots, r$ and $x_{i j} \in V_{i j}, i<j$.

In what follows, we introduce the definition of another central concept used in this paper and that is the definition of the symmetric cone and its relation to EJA.

A non-empty subset $\mathcal{K}$ of $V$ is a cone iff $x \in \mathcal{K}$ and $\lambda>0$ imply that $\lambda x \in \mathcal{K}$. An interior of a cone $\mathcal{K}$ is denoted as $\mathcal{K}^{0}$. The dual cone of $\mathcal{K}$ is defined as the set

$$
\mathcal{K}^{*}:=\{y \in V:\langle x, y\rangle \geq 0, \forall x \in \mathcal{K}\} .
$$

It is easy to show that $\mathcal{K}^{*}$ is a closed and convex cone. A cone is self-dual iff $\mathcal{K}=\mathcal{K}^{*}$ and it is pointed iff $\mathcal{K} \cap-\mathcal{K}=\{0\}$. In what follows, $\mathcal{K}$ will denote a non-empty, pointed and convex cone.

For a convex cone $\mathcal{K}$ we define the automorphism group, Aut $(\mathcal{K}) \subset \mathrm{GL}(\mathrm{V})$ of $\mathcal{K}$ by

$$
\operatorname{Aut}(\mathcal{K}):=\{g \in \mathrm{GL}(\mathrm{V}): g(\mathcal{K})=\mathcal{K}\},
$$

where GL $(V)$ is the set of invertible linear maps $g$ from $V$ into itself.

The convex cone $\mathcal{K}$ is said to be homogeneous if $\operatorname{Aut}(\mathcal{K})$ acts transitively on $\mathcal{K}^{0}$, i.e., for all $x, y \in \mathcal{K}^{0}$ there exists $g \in \operatorname{Aut}(\mathcal{K})$ such that $g x=y$. The convex cone $\mathcal{K}$ is said to be symmetric iff it is homogeneous and self-dual.

In the framework of EJA a very important fact has been observed.

Theorem 2.3 (Theorem III.3.1 in [2]) A cone $\mathcal{K}$ is symmetric iff it is a cone of squares of some Euclidean Jordan algebra $V$, i.e.

$$
\mathcal{K}=\mathcal{K}(V):=\left\{x^{2}: x \in V\right\} .
$$

The reason why symmetric cones on EJAs serve as a unifying framework in optimization is the fact that important cones used in optimization such as the non-negative orthant, the second order cone, and the semidefinite cone are all examples of symmetric cones on EJAs.

It is worth mentioning that for $x \in \mathcal{K}$ the corresponding eigenvalues are nonnegative, i.e. $\lambda_{i}(x) \geq 0, i=1, \ldots, r$. Furthermore, if $x$ is in the interior of the cone, $x \in \mathcal{K}^{0}$, then the corresponding eigenvalues are strictly positive. Moreover, if $x \in \mathcal{K}$, $y \in \mathcal{K}$, then $\operatorname{tr}(x \circ y) \geq 0$, which is by (5) equivalent to $\langle x, y\rangle \geq 0$. Also, it can be shown (Lemma 2.2 in [7]) that

$$
\langle x, y\rangle=0 \quad \Leftrightarrow \quad x \circ y=0,
$$

which is of crucial importance in the design of the IPMs in the framework of EJA.

In the sequel, we will need also a concept of similarity of two elements of EJA. Two elements $x, y \in V$ are said to be similar, with notation $x \sim y$, if and only if 
they share the same eigenvalues, including their multiplicities. This is equivalent to the similarity of their linear representations (Proposition 19 in [10]). If, in addition, $x, y \in \mathcal{K}^{0}$, then their quadratic representations are also similar (Corollary 20 in [10]). Thus, for $x, y \in \mathcal{K}^{0}$ we can write

$$
x \sim y \quad \Leftrightarrow \quad L(x) \sim L(y) \quad \Leftrightarrow \quad P(x) \sim P(y) .
$$

Another important building block, which we need in the sequel, is the concept of various types of functions on EJA and the corresponding symmetric cone. The functions that we will use in this paper are separable spectral functions induced by univariate functions as described below.

Let $f(\cdot)$ be univariate function and $x=\sum_{i=1}^{r} \lambda_{i}(x) c_{i}$ be a unique spectral decomposition of $x \in V$. Then we define $F: V \rightarrow \mathbf{R}$ by

$$
F(x):=\sum_{i=1}^{r} f\left(\lambda_{i}(x)\right)
$$

and $G: V \rightarrow V$ by

$$
G(x):=\sum_{i=1}^{r} f\left(\lambda_{i}(x)\right) c_{i} .
$$

The first derivative $D_{x} F(x)$ of the function $F$, or as it is also called, the gradient $\nabla F(x)$ is given in the following theorem.

Theorem 2.4 (Theorem 3.6.2 in [11]) Let $D \subset \mathbf{R}$ be an open set and $f: D \mapsto \mathbf{R}$. Let $U=\left\{x \in V: \lambda(x) \in D^{r}\right\}$ and $F: U \mapsto \mathbf{R}$ defined as $F(x)=\sum_{i=1}^{r} f\left(\lambda_{i}(x)\right)$, with $x=\sum_{i=1}^{r} \lambda_{i}(x) c_{i} \in V$. If $f$ is differentiable in $D$, then $F$ is differentiable in $U$ and

$$
\nabla F(x)=D_{x} F(x)=\sum_{i=1}^{r} f^{\prime}\left(\lambda_{i}(x)\right) c_{i} .
$$

The first derivative of the function $G$ is given in the following theorem.

Theorem 2.5 (Lemma 3.6.3 in [11]) If $f$ is a continuously differentiable function in a suitable domain that contains all the eigenvalues of $x \in V$, then $G(x)=$ $\sum_{i=1}^{r} f\left(\lambda_{i}(x)\right) c_{i}$ is continuously differentiable at $x$ and

$$
D_{x} G(x)=\sum_{i=1}^{r} f^{\prime}\left(\lambda_{i}\right) P_{i i}+\sum_{j<k} \frac{f\left(\lambda_{j}\right)-f\left(\lambda_{k}\right)}{\lambda_{j}-\lambda_{k}} P_{j k},
$$

with $P_{j k}, 1 \leq j \leq k \leq r$ as defined in Theorem 2.2.

The above results, as well as a more general treatment of spectral functions, their derivatives and various properties is partially based on considering eigenvalues of $x$ as functions, $\lambda_{i}: V \rightarrow \mathbf{R}$, and calculating their derivatives. Details can be found in $[2,11,29]$. 


\section{Interior Point Method for SCLCP}

We consider the monotone SCLCP (1) in the standard form: Given an $n$-dimensional $\operatorname{EJA}(V, \circ)$ and its associated symmetric cone of squares $\mathcal{K}$, find $(x, s) \in \mathcal{K} \times \mathcal{K}$ such that

$$
s=M x+q, \quad x \circ s=0,
$$

where $M \in \mathbf{R}^{n \times n}$ and $q \in \mathbf{R}^{n}$. The first equation is a standard representation of the affine subspace $\mathcal{L}$ and the second equation is due to (10).

It is well known that SCLCP is an NP-hard problem for a general matrix $M$, even in the case when $\mathcal{K}=\mathbf{R}_{+}^{n}$ (see [30]). Thus, we need to restrict ourselves to classes of matrices for which polynomial IPMs exist. The class of monotone matrices appears most frequently. The monotone property can be formulated as follows:

$$
v=M u \quad \Rightarrow \quad\langle u, v\rangle \geq 0
$$

where the inner product is defined by (5). This is equivalent with the fact that matrix $M$ is positive semidefinite with respect to the inner product $\langle\cdot, \cdot\rangle$ in $(V, \circ)$.

Furthermore, we assume that the above SCLCP satisfy the interior-point condition (IPC), that is, there exists a point $(x, s) \in \mathcal{K}^{0} \times \mathcal{K}^{0}$ such that $s=M x+q$ and $\mathcal{K}^{0}$ represents the interior of $\mathcal{K}$.

In what follows we outline the main idea of the kernel-based interior-point method (IPM) for the above SCLCP (13). The standard approach is to consider the perturbed system

$$
\begin{aligned}
s & =M x+q, \\
x \circ s & =\mu e, \\
(x, s) & \in \mathcal{K}^{0} \times \mathcal{K}^{0} .
\end{aligned}
$$

Given the assumptions, the above system has a unique solution, for each $\mu>0$. This solution is denoted as $(x(\mu), s(\mu))$ and we call it the $\mu$-center of SCLCP. The set of $\mu$-centers gives a path, which is called the central path of SCLCP [8]. Hence, if $\mu \rightarrow 0$, the limit of the central path exists and it is a solution of SCLCP.

The limiting property of the central path, mentioned above, leads naturally to the main idea of the IPMs for solving SCLCP: trace the central path while reducing $\mu$ at each iteration. However, tracing the central path exactly would be too costly and inefficient. It has been shown that it is sufficient to trace the central path approximately.

Suppose that a strictly feasible point $(x, s)$ that is 'close' to the $\mu$-center $(x(\mu), s(\mu))$, for some positive $\mu$, is known. The 'closeness' is measured using a barrier function, as discussed later in the paper. We then decrease $\mu$ to $\mu_{+}:=(1-\theta) \mu$, for some $\theta \in] 0,1\left[\right.$ and, redefining $\mu:=\mu_{+}$calculate a search direction using Newton's method.

$$
\begin{aligned}
-M \Delta x+\Delta s & =0, \\
s \circ \Delta x+x \circ \Delta s & =-x \circ s+\mu e .
\end{aligned}
$$


As it was first discovered for Semidefinite Optimization (SDO) the above system unfortunately does not have a unique solution. In order to fix this problem a scaling scheme had to be applied. Several different scaling schemes were proposed [5, 22, 31, 32] leading to different search directions. Monteiro and Zhang [33] showed that they all belong to the so-called Monteiro-Zhang class of scaling schemes (directions). The generalization to the symmetric cones case was first proposed by Faybusovich $[7,8]$ and then further discussed by Alizadeh and Schmieta $[9,10]$.

It has been shown that the reason why the system (16) does not have a unique solution is the fact that $x$ and $s$ do not operator commute in general, that is,

$$
L(x) L(s) \neq L(s) L(x) \text {. }
$$

The scaling scheme is based on the following fact (Lemma 28 in [10]): Let $u \in \mathcal{K}^{0}$. Then,

$$
x \circ s=\mu e \quad \Leftrightarrow \quad P(u) x \circ P\left(u^{-1}\right) s=\mu e .
$$

Now replacing the second equation of (15) by $P(u) x \circ P\left(u^{-1}\right) s=\mu e$ and applying Newton's method we obtain the system

$$
\begin{aligned}
-M \Delta x+\Delta s & =0, \\
P\left(u^{-1}\right) s \circ P(u) \Delta x+P(u) x \circ P\left(u^{-1}\right) \Delta s & =-P(u) x \circ P\left(u^{-1}\right) s+\mu e .
\end{aligned}
$$

The appropriate choices of $u$ that lead to obtaining unique search directions from system (17) can be generalized from the SDO case. Muramatsu [34] proposed choices of $u$ that define a wide commutative class of search directions that includes the Monteiro-Zhang class of directions. In this paper we will use the classical NesterovTodd scaling point to find unique Nesterov-Todd direction (NT-direction)

$$
u=w^{-\frac{1}{2}}, \quad w=\left(P(x)^{\frac{1}{2}}\right)\left(P\left(x^{\frac{1}{2}}\right) s\right)^{-\frac{1}{2}} \quad\left[=P\left(s^{-\frac{1}{2}}\right)\left(P\left(s^{\frac{1}{2}}\right) x\right)^{\frac{1}{2}}\right] .
$$

This scaling point was first proposed by Nesterov and Todd for self-scaled cones [5] and then adapted by Faybusovich [8] for symmetric cones.

A few brief remarks are in order. First, it is worth mentioning that for given $(x, s) \in$ $\mathcal{K}^{0} \times \mathcal{K}^{0}, w \in \mathcal{K}^{0}$ is the unique scaling point such that $P(w) s=x$ (Lemma 2.4 in [8] or Proposition 5.4.2 in [11]). Second, another way to look at $w$ is to observe that $w$ is precisely the geometric mean of $x$ and $s^{-1}$, where geometric mean of $a, b \in \mathcal{K}^{0}$ is defined as

$$
a \# b:=P\left(a^{\frac{1}{2}}\right)\left(P\left(a^{-\frac{1}{2}}\right) b\right)^{\frac{1}{2}} .
$$

Thus, $w=x \# s^{-1}$. The geometric mean and its properties have been studied by Lim [35].

For the formulation and analysis of the generic IPM for SCLCP that we want to present, the introduction of the following variance vector is critical:

$$
v:=\frac{1}{\sqrt{\mu}} P(w)^{-\frac{1}{2}} x \quad\left[=\frac{1}{\sqrt{\mu}} P(w)^{\frac{1}{2}} s\right] .
$$


Note that (Proposition 5.7.2 in [11])

$$
x \circ s=\mu e \Leftrightarrow v=e .
$$

Using the variance vector $v$ the following scaled search directions are introduced:

$$
d_{x}=\frac{1}{\sqrt{\mu}} P(w)^{-\frac{1}{2}} \Delta x, \quad d_{s}=\frac{1}{\sqrt{\mu}} P(w)^{\frac{1}{2}} \Delta s .
$$

Using (22) the system (17) can be rewritten as

$$
\begin{aligned}
-\bar{M} d_{x}+d_{s} & =0, \\
d_{x}+d_{s} & =v^{-1}-v,
\end{aligned}
$$

where

$$
\bar{M}:=P(w)^{\frac{1}{2}} M P(w)^{\frac{1}{2}} .
$$

It is obvious that if $M$ is positive semidefinite matrix, then $\bar{M}$ is also positive semidefinite:

$$
\begin{aligned}
\langle u, \bar{M} u\rangle & =\left\langle u,\left(P(w)^{\frac{1}{2}} M P(w)^{\frac{1}{2}}\right) u\right\rangle \\
& =\left\langle P(w)^{\frac{1}{2}} u, M\left(P(w)^{\frac{1}{2}} u\right)\right\rangle \geq 0 .
\end{aligned}
$$

Given the spectral decomposition of $v$ with respect to the Jordan frame $\left(c_{1}, \ldots, c_{r}\right)$, $v=\sum_{i=1}^{r} \lambda_{i}(v) c_{i}$, the scaled logarithmic barrier function is defined as

$$
\Psi_{c}(v):=\sum_{i=1}^{r}\left(\frac{\lambda_{i}(v)^{2}-1}{2}-\log \lambda_{i}(v)\right) .
$$

A crucial observation is that the second equation in (23) can now be written as

$$
v^{-1}-v=-\nabla \Psi_{c}(v)
$$

where $\nabla \Psi_{c}(v)$ is defined as described in Theorem 2.4. This means that the logarithmic barrier function essentially determines the search direction.

Another important observation is that since $\nabla \Psi_{c}(e)=0$, it follows that $\Psi_{c}(v)$ attains its minimal value at $v=e$, with $\Psi_{c}(e)=0$, that is, according to (21), if and only if $x=x(\mu)$ and $s=s(\mu)$, implying that the minimizer of $\Psi_{c}(v)$ is the $\mu$-center. In addition, $\Psi_{c}(v)$ is strictly convex function. The implication is that the function $\Psi_{c}(v)$ essentially serves as a 'proximity' measure of closeness for $(x, s)$ with respect to the $\mu$-center.

Our generalization follows the argument that $\Psi_{c}(v)$ can be replaced by any function $\Psi(v), v \in \mathcal{K}^{0}$, with the same properties as the scaled logarithmic barrier function (24), that is, $\Psi(v)$ is strictly convex and attains its minimum at $v=e$ with $\Psi(e)=0$. The function $\Psi(v)$ is called a scaled barrier function. Since $\Psi(v)=0 \Leftrightarrow$ $\nabla \Psi(v)=0 \Leftrightarrow v=e$, the function $\Psi(v)$ still serves as a proximity measure to the $\mu$ center. Specifically, the inequality $\Psi(v) \leq \tau$ with $\tau>0$ as a threshold value defines 


\section{Generic IPM for SCLCP}

\section{Input:}

A threshold parameter $\tau \geq 1$;

an accuracy parameter $\varepsilon>0$;

a fixed barrier update parameter $\theta 0<\theta<1$;

a starting point $\left(x^{0}, s^{0}\right)$ with $\mu^{0}=\left(x^{0}\right)^{T} s^{0} / n$ such that $\Psi\left(v^{0}\right) \leq \tau$, where $v^{0}$ is calculated using (18) and (20).

\section{begin}

$x:=x^{0} ; s:=s^{0} ; \mu:=\mu^{0}$,

while $n \mu \geq \varepsilon$ do

begin

$\mu:=(1-\theta) \mu$;

calculate $v$ using (18) and (20);

while $\Psi(v)>\tau$ do

begin

calculate search direction $(\Delta x, \Delta s)$;

determine the step size $\alpha$;

update $(x, s):=(x, s)+\alpha(\Delta x, \Delta s)$.

end

end

end

Fig. 1 Generic IPM for SCLCP

a $\tau$-neighborhood of the $\mu$-center. In addition, the following norm-based proximity measure will play an important role in the analysis of the algorithm:

$$
\delta(v):=\frac{1}{2}\|\nabla \Psi(v)\|
$$

The scaled centering equation in (23) becomes $d_{x}+d_{s}=-\nabla \Psi(v)$ and, hence, the search direction is calculated from the following system

$$
\begin{aligned}
-\bar{M} d_{x}+d_{s} & =0, \\
d_{x}+d_{s} & =-\nabla \Psi(v) .
\end{aligned}
$$

The above discussion can be summarized in the form of the Generic IPM presented in Fig. 1.

In what follows we will refer to the Generic IPM for SCLCP simply as the Generic Algorithm. The behavior of the Generic Algorithm depends on the choice of the values for the input parameters as well as the choice of the barrier function and the step size. It is now standard to call IPM a large-update method if $\theta$ is a fixed constant, and $\theta \in] 0,1[$ is independent of the dimension $n$ of the problem, i.e., $\theta=O(1)$ 
and $\tau=O(n)$. If $\theta$ depends on the dimension of the problem with $\theta=O\left(\frac{1}{\sqrt{n}}\right)$ and $\tau=O(1)$, IPM is called a small-update method.

The goal is to choose the values of the parameters in such a way that the number of iterations required by the Generic Algorithm is as small as possible.

\section{Kernel-Based Barrier Functions}

As already observed in the previous section, the behavior of the Generic Algorithm depends heavily on the choice of the barrier function.

We can look at the log-barrier function defined in (24) from another angle by introducing the univariate function $\left.\psi_{c}:\right] 0, \infty[\rightarrow[0, \infty[$ defined as

$$
\psi_{c}(t)=\frac{t^{2}-1}{2}-\log t
$$

Then $\Psi_{c}(v)$ can be written as

$$
\Psi_{c}(v)=\sum_{i=1}^{n} \psi_{c}\left(\lambda_{i}(v)\right),
$$

that is, as a separable function with identical univariate functions for each component. The univariate function $\psi_{c}$ is called the logarithmic kernel function.

We consider barrier functions of the same type as the logarithmic barrier function, that is, separable functions

$$
\Psi(v):=\sum_{i=1}^{n} \psi\left(\lambda_{i}(v)\right),
$$

defined by the univariate function $\psi:] 0, \infty[\rightarrow[0, \infty[$, called the kernel function. We require that $\psi$ has the same properties as the logarithmic kernel function, that is,

$$
\begin{aligned}
\psi^{\prime}(1)=\psi(1) & =0, \\
\psi^{\prime \prime}(t) & >0, \\
\lim _{t \downarrow 0} \psi(t)=\lim _{t \rightarrow \infty} \psi(t) & =\infty .
\end{aligned}
$$

These properties have some important implications: Clearly, (30a) and (30b) say that $\psi(t)$ is a non-negative strictly convex function such that it achieves its minimum at $t=1$, i.e., $\psi(1)=0$. Moreover, (30c) expresses that $\psi(t)$ is coercive and has the barrier property. Furthermore, the conditions imply that $\psi(t)$ is decreasing for $0<t<1$ and increasing for $t>1$. A consequence of (30b) is that $\psi^{\prime}(t)$ is increasing for $t>0$. Note that the conditions imply that $\psi(t)$ is completely determined by its second derivative:

$$
\psi(t)=\int_{1}^{t} \int_{1}^{\xi} \psi^{\prime \prime}(\zeta) d \zeta d \xi
$$


In order to obtain favorable iterations bounds we introduce the following additional conditions on the kernel function:

$$
\begin{aligned}
t \psi^{\prime \prime}(t)+\psi^{\prime}(t) & >0, & t<1, \\
\psi^{\prime \prime \prime}(t) & <0, & t>0, \\
2 \psi^{\prime \prime}(t)^{2}-\psi^{\prime}(t) \psi^{\prime \prime \prime}(t) & >0, & t<1, \\
\psi^{\prime \prime}(t) \psi^{\prime}(\beta t)-\beta \psi^{\prime}(t) \psi^{\prime \prime}(\beta t) & >0, & t>1, \beta>1 .
\end{aligned}
$$

The kernel functions that satisfy conditions (30a)-(30c) and (32a)-(32d) are called eligible kernel functions. They were first introduced for LO by Bai et al. in [28] and revisited for $P_{*}(\kappa)$-LCP over non-negative orthant in $[1,15]$.

The following remark is in order: in [28] another condition is also introduced, that is,

$$
t \psi^{\prime \prime}(t)-\psi^{\prime}(t)>0, \quad t>1 .
$$

This condition is listed because conditions (33) and (32b) imply condition (32d) (Lemma 4.4 in [28]). It is easier to check condition (33) than (32d) which is more technically involved. Many eligible functions do satisfy condition (33). Thus, the kernel function is also eligible if it satisfies conditions (32a)-(32c), and (33). It is also shown in [28] that the conditions (32a)-(32c), and (33) are logically independent.

One of the essential properties of eligible kernel functions is property (ii) in the lemma below. We call this property the exponential-convexity property.

Lemma 4.1 (Lemma 2.1.2 in [36]) Let $\psi(t)$ be a twice differentiable function for $t>0$. Then the following three properties are equivalent:

(i) $\psi\left(\sqrt{t_{1} t_{2}}\right) \leq \frac{1}{2}\left(\psi\left(t_{1}\right)+\psi\left(t_{2}\right)\right)$, for $t_{1}, t_{2}>0$;

(ii) $\psi^{\prime}(t)+t \psi^{\prime \prime}(t) \geq 0, t>0$;

(iii) $\psi\left(e^{\xi}\right)$ is convex.

The above listed exponential convexity of a kernel function implies the exponential convexity of the associated barrier function which is crucial in the subsequent analysis of the Generic Algorithm.

Theorem 4.1 (Theorem 4.3.2 in [11]) Let $\Psi(v)$ be the function defined in (29) and $x, s \in \mathcal{K}^{0}$. Then

$$
\Psi\left(\left(P(x)^{1 / 2} s\right)^{1 / 2}\right) \leq \frac{1}{2}(\Psi(x)+\Psi(s)) .
$$

In the analysis of the algorithm we will need a notion of the first and second directional derivatives of the barrier function and the upper bound on the second derivative.

Let $x(t):=x_{0}+t u$ with $t \in \mathbf{R}$ and $u \in V$ and assume that $x(t) \in \mathcal{K}^{0}$. Let $x(t)=$ $\sum_{i=1}^{r} \lambda_{i}(x(t)) c_{i}$ be the spectral decomposition of $x(t)$ with respect to the Jordan 
frame $c_{1}, \ldots, c_{r}$. Using Theorem 2.4 we have

$$
D_{t} \Psi(x(t))=\operatorname{tr}\left(D_{x} \Psi(x(t)) \circ x^{\prime}(t)\right)=\operatorname{tr}\left(\sum_{i=1}^{r} \psi^{\prime}\left(\lambda_{i}(x(t))\right) c_{i} \circ u\right),
$$

and using Theorem 2.5 we obtain

$$
\begin{aligned}
D_{t}^{2} \Psi(x(t))= & \sum_{i=1}^{r} \psi^{\prime \prime}\left(\lambda_{i}\right) u_{i}^{2}+\sum_{j<k, \lambda_{j}=\lambda_{k}} \psi^{\prime \prime}\left(\lambda_{j}\right) \operatorname{tr}\left(u_{j k}^{2}\right) \\
& +\sum_{j<k, \lambda_{j} \neq \lambda_{k}} \frac{\psi^{\prime}\left(\lambda_{j}\right)-\psi^{\prime}\left(\lambda_{k}\right)}{\lambda_{j}-\lambda_{k}} \operatorname{tr}\left(u_{j k}^{2}\right),
\end{aligned}
$$

where

$$
u=\sum_{i=1}^{r} u_{i} c_{i}+\sum_{i<j} u_{i j}
$$

is the Peirce decomposition of $u$ with respect to the Jordan frame $c_{1}, \ldots, c_{r}$. The details of the derivation can be found in Sect. 4.4 of [11].

Furthermore an upper bound on the second derivative is needed in the sequel and is given by the following lemma.

Lemma 4.2 (Lemma 4.4.1 in [11]) One has

$$
D_{t}^{2} \Psi(x(t)) \leq \sum_{i=1}^{r} \psi^{\prime \prime}\left(\lambda_{i}\right) u_{i}^{2}+\sum_{j<k} \psi^{\prime \prime}\left(\lambda_{k}\right) \operatorname{tr}\left(u_{j k}^{2}\right)
$$

Two inverse functions related to the kernel function have shown to be essential in the analysis of the Generic Algorithm and are defined below.

Definition 4.1 Given the kernel function $\psi$, we define

(i) $\varrho:[0, \infty[\rightarrow[1, \infty[$ is the inverse function of $\psi(t)$ for $t \geq 1$;

(ii) $\rho:[0, \infty[\rightarrow] 0,1]$ is the inverse function of $-\frac{1}{2} \psi^{\prime}(t)$ for $t \leq 1$.

It is worth mentioning that $\varrho$ is an increasing function since $\psi(t)$ for $t \geq 1$ is an increasing function because of its definition, while $\rho$ is a decreasing function because $\psi^{\prime}(t)$ is increasing for $t>0$ due to $\psi^{\prime \prime}(t)>0$ and therefore $-\frac{1}{2} \psi^{\prime}(t)$ for $t \leq 1$ is decreasing.

In Table 1 we list ten frequently used eligible functions.

A few brief comments about the listed kernel functions are in order. Function $\psi_{1}$ is the classical logarithmic kernel function. Function $\psi_{7}$ is the prototype self-regular kernel function introduced by Peng et al. in [36]. Function $\psi_{4}$ is actually function $\psi_{7}$ with $q=3$. Function $\psi_{8}$ is not self-regular; it has a linear growth term and was considered first in [37]. Function $\psi_{10}$ includes functions $\psi_{1}, \psi_{4}, \psi_{7}, \psi_{8}$ and $\psi_{9}$ as special cases. It was first considered in [38]. 
Table 1 Ten eligible kernel functions

\begin{tabular}{ll}
\hline$i$ & Kernel functions $\psi_{i}$ \\
\hline 1 & $\frac{t^{2}-1}{2}-\log t$ \\
2 & $\frac{t^{2}-1}{2}+\frac{t^{1-q}-1}{q(q-1)}-\frac{q-1}{q}(t-1), q>1$ \\
3 & $\frac{t^{2}-1}{2}+\frac{(e-1)^{2}}{e} \frac{1}{e^{t}-1}-\frac{e-1}{e}$ \\
4 & $\frac{1}{2}\left(t-\frac{1}{t}\right)^{2}$ \\
5 & $\frac{t^{2}-1}{2}+e^{\frac{1}{t}-1}-1$ \\
6 & $\frac{t^{2}-1}{2}-\int_{1}^{t} e^{\frac{1}{\xi}-1} d \xi$ \\
7 & $\frac{t^{2}-1}{2}+\frac{t^{1-q}-1}{q-1}, q>1$ \\
8 & $t-1+\frac{t^{1-q}-1}{q-1}, q>1$ \\
9 & $\frac{t^{1+p}-1}{1+p}-\log t, p \in[0,1]$ \\
10 & $\frac{t^{p+1}-1}{p+1}+\frac{t^{1-q}-1}{q-1}, p \in[0,1], q>1$ \\
\hline
\end{tabular}

\section{Analysis of the Algorithm}

The analysis of the Generic Algorithms that is presented in the sequel follows the same outline employed in papers on IPMs with eligible kernel functions for LO [28] and for LCP [1]) over the non-negative orthant. In addition, the analysis builds on the only work on IPM with eligible kernel functions for LO over symmetric cones [11].

\subsection{Growth of the Barrier Function During an Outer Iteration}

At the start of each outer iteration, just before the update of $\mu$ with the factor $1-\theta$, we have $\Psi(v) \leq \tau$. Due to the update of $\mu$ the vector $v$ is divided by the factor $\sqrt{1-\theta}$, with $0<\theta<1$, which in general leads to an increase in the value of $\Psi(v)$. It is important to estimate that increase.

The result is based on the following theorem which first appeared in [28]. The version of the theorem for LO over symmetric cones was first proved in [11] and can be adopted here without major changes. Furthermore, it is worth noting that in its proof condition $(32 \mathrm{~d})$ is used.

Theorem 5.1 (Theorem 5.9.1 in [11]) Let $\varrho:[0, \infty[\rightarrow[1, \infty[$ be the inverse function of the kernel function $\psi(t)$ for $t \geq 1$. Then, for any vector $v \in \mathcal{K}^{0}$ and any $\beta \geq 1$ the following inequality holds:

$$
\Psi(\beta v) \leq r \psi\left(\beta \varrho\left(\frac{\Psi(v)}{r}\right)\right) .
$$

Corollary 5.1 Let $0 \leq \theta \leq 1$ and $v_{+}=\frac{v}{\sqrt{1-\theta}}$. If $\Psi(v) \leq \tau$, then

$$
\Psi\left(v_{+}\right) \leq r \psi\left(\frac{\varrho\left(\frac{\tau}{r}\right)}{\sqrt{1-\theta}}\right) \leq \frac{r}{2} \psi^{\prime \prime}(1)\left(\frac{\varrho\left(\frac{\tau}{r}\right)}{\sqrt{1-\theta}}-1\right)^{2} .
$$




\subsection{Determining the Default Step Size}

The goal is to compute a default value for the step size $\alpha$ in order to yield a new pair $\left(x_{+}, s_{+}\right)$that will result in a sufficient decrease of the barrier function during an inner iteration.

After a damped step, we have

$$
\begin{aligned}
x_{+} & :=x+\alpha \Delta x=x+\sqrt{\mu} \alpha P(w)^{\frac{1}{2}} d_{x} \\
& =P(w)^{\frac{1}{2}}\left(P(w)^{-\frac{1}{2}} x+\sqrt{\mu} \alpha d_{x}\right) \\
& =P(w)^{\frac{1}{2}}\left(\sqrt{\mu} v+\sqrt{\mu} \alpha d_{x}\right),
\end{aligned}
$$

and we get

$$
x_{+}=\sqrt{\mu} P(w)^{\frac{1}{2}}\left(v+\alpha d_{x}\right) .
$$

Similarly, we obtain

$$
s_{+}=s+\alpha \Delta s=\sqrt{\mu} P(w)^{-\frac{1}{2}}\left(v+\alpha d_{s}\right) .
$$

Thus,

$$
v_{+}:=\frac{1}{\sqrt{\mu}} P\left(w_{+}\right)^{-\frac{1}{2}} x_{+}=\frac{1}{\sqrt{\mu}} P\left(w_{+}\right)^{\frac{1}{2}} s_{+},
$$

or equivalently

$$
v_{+}=P\left(w_{+}\right)^{\frac{1}{2}} P(w)^{-\frac{1}{2}}\left(v+\alpha d_{s}\right)=P\left(w_{+}\right)^{-\frac{1}{2}} P(w)^{\frac{1}{2}}\left(v+\alpha d_{x}\right),
$$

where, according to (19),

$$
w_{+}:=P\left(x_{+}\right)^{\frac{1}{2}}\left(\left(P\left(x_{+}\right)^{\frac{1}{2}} s_{+}\right)^{-\frac{1}{2}}\right) .
$$

We want to calculate the decrease of the barrier function $\Psi(v)$ and we consider the decrease as a function of $\alpha$, that is, we define

$$
f(\alpha):=\Psi\left(v_{+}\right)-\Psi(v) .
$$

However, working with $f(\alpha)$ may not be easy because in general $f(\alpha)$ is not convex. Thus, we are searching for a convex function $f_{1}(\alpha)$ that is an upper bound of $f(\alpha)$ and whose derivatives are easier to calculate than those of $f(\alpha)$. The key element in this process is replacing $v_{+}$with a similar element, which will allow the use of exponential convexity of the barrier function. By Proposition 5.9.3 in [11] we have

$$
v_{+} \sim\left(P\left(v+\alpha d_{x}\right)^{\frac{1}{2}}\left(v+\alpha d_{s}\right)\right)^{\frac{1}{2}}
$$

and therefore

$$
\Psi\left(v_{+}\right)=\Psi\left(\left(P\left(v+\alpha d_{x}\right)^{\frac{1}{2}}\left(v+\alpha d_{s}\right)\right)^{\frac{1}{2}}\right) .
$$


Now using Theorem 4.1 we obtain

$$
\Psi\left(v_{+}\right) \leq \frac{1}{2}\left(\Psi\left(v+\alpha d_{x}\right)+\Psi\left(v+\alpha d_{s}\right)\right) .
$$

Defining

$$
f_{1}(\alpha):=\frac{1}{2}\left(\Psi\left(v+\alpha d_{x}\right)+\Psi\left(v+\alpha d_{s}\right)\right)-\Psi(v)
$$

we easily see that

$$
f(\alpha) \leq f_{1}(\alpha)
$$

Obviously,

$$
f(0)=f_{1}(0)=0 .
$$

In what follows we will examine the derivatives of $f_{1}(\alpha)$ and show that the function is convex in $\alpha$.

Using the definition of the derivative of the barrier function (35), the derivative of $f_{1}(\alpha)$ with respect to $\alpha$ is given by

$$
f_{1}^{\prime}(\alpha)=\frac{1}{2}\left(\operatorname{tr}\left(\Psi^{\prime}\left(v+\alpha d_{x}\right) \circ d_{x}\right)+\operatorname{tr}\left(\Psi^{\prime}\left(v+\alpha d_{s}\right) \circ d_{s}\right)\right) .
$$

Particularly,

$$
\begin{aligned}
f_{1}^{\prime}(0) & =\frac{1}{2} \operatorname{tr}\left(\Psi^{\prime}(v) \circ\left(d_{x}+d_{s}\right)\right)=-\frac{1}{2} \operatorname{tr}(\nabla \Psi(v) \circ \nabla \Psi(v)) \\
& =-\frac{1}{2}\|\nabla \Psi(v)\|^{2}=-2 \delta^{2}(v),
\end{aligned}
$$

which implies that $f_{1}(\alpha)$ is monotonically decreasing in the neighborhood of $\alpha=0$.

Next, we examine $f_{1}^{\prime \prime}(\alpha)$. Using the spectral decompositions we can write

$$
v+\alpha d_{x}=\sum_{i=1}^{r} \lambda_{i}\left(v+\alpha d_{x}\right) c_{i}, \quad v+\alpha d_{s}=\sum_{i=1}^{r} \lambda_{i}\left(v+\alpha d_{s}\right) b_{i} .
$$

Let

$$
d_{x}=\sum_{i=1}^{r} d_{x i} c_{i}+\sum_{i<j} d_{x i j},
$$

be the Peirce decompositions of $d_{x}$ with respect to the Jordan frame $c_{1}, \ldots, c_{r}$ and

$$
d_{s}=\sum_{i=1}^{r} d_{s i} b_{i}+\sum_{i<j} d_{s i j}
$$

be the Peirce decomposition of $d_{s}$ with respect to the Jordan frame $b_{1}, \ldots, b_{r}$. For simplicity of notation, below we use

$$
\eta_{i}:=\lambda_{i}\left(v+\alpha d_{x}\right), \quad \gamma_{i}=\lambda_{i}\left(v+\alpha d_{s}\right), \quad i=1, \ldots, r .
$$


Thus, if we differentiate $f_{1}^{\prime}$ with respect to $\alpha$, using (36), we obtain

$$
f_{1}^{\prime \prime}(\alpha)=g_{1}(\alpha)+g_{2}(\alpha),
$$

where

$$
\begin{aligned}
g_{1}(\alpha)= & \sum_{i=1}^{r} \psi^{\prime \prime}\left(\eta_{i}\right) d_{x_{i}}^{2}+\sum_{i<j, \eta_{i}=\eta_{j}} \psi^{\prime \prime}\left(\eta_{i}\right) \operatorname{tr}\left(d_{x i j}^{2}\right) \\
& +\sum_{i<j, \eta_{i} \neq \eta_{j}} \frac{\psi^{\prime}\left(\eta_{i}\right)-\psi^{\prime}\left(\eta_{j}\right)}{\eta_{i}-\eta_{j}} \operatorname{tr}\left(d_{x_{i j}}^{2}\right),
\end{aligned}
$$

and

$$
\begin{aligned}
g_{2}(\alpha)= & \sum_{i=1}^{r} \psi^{\prime \prime}\left(\gamma_{i}\right) d_{s i}^{2}+\sum_{i<j, \gamma_{i}=\gamma_{j}} \psi^{\prime \prime}\left(\gamma_{i}\right) \operatorname{tr}\left(d_{s i j}^{2}\right) \\
& +\sum_{i<j, \gamma_{i} \neq \gamma_{j}} \frac{\psi^{\prime}\left(\gamma_{i}\right)-\psi^{\prime}\left(\gamma_{j}\right)}{\gamma_{i}-\gamma_{j}} \operatorname{tr}\left(d_{s i j}^{2}\right) .
\end{aligned}
$$

Now, using Lemma 4.2 we obtain the following upper bound on $f_{1}^{\prime \prime}(\alpha)$ :

$$
\begin{aligned}
f_{1}^{\prime \prime}(\alpha) \leq & \frac{1}{2}\left(\sum_{i=1}^{r} \psi^{\prime \prime}\left(\eta_{i}\right) d_{x_{i}}^{2}+\sum_{i<j} \psi^{\prime \prime}\left(\eta_{j}\right) \operatorname{tr}\left(d_{x_{i j}}^{2}\right)\right) \\
& +\frac{1}{2}\left(\sum_{i=1}^{r} \psi^{\prime \prime}\left(\gamma_{i}\right) d_{s i}^{2}+\sum_{i<j} \psi^{\prime \prime}\left(\gamma_{j}\right) \operatorname{tr}\left(d_{s i j}^{2}\right)\right) .
\end{aligned}
$$

The key steps in the analysis are based on the effort to find an upper bound on $\left\|d_{x}\right\|$ and $\left\|d_{s}\right\|$ in terms of the proximity measure $\delta$. Contrary to the LO case the vectors $d_{x}$ and $d_{s}$ are not necessarily orthogonal any more. However, the monotonicity of the SCLCP still allows us to find a good bound.

Lemma 5.1 For search directions $d_{x}, d_{s}$ obtained in (22) the following upper bounds hold:

$$
\left\|d_{x}\right\| \leq 2 \delta \text { and } \quad\left\|d_{s}\right\| \leq 2 \delta .
$$

Proof We have

$$
\begin{aligned}
\left\|d_{x}+d_{s}\right\|^{2} & =\left\langle d_{x}+d_{s}, d_{x}+d_{s}\right\rangle=\left\|d_{x}\right\|^{2}+2\left\langle d_{x}, d_{s}\right\rangle+\left\|d_{s}\right\|^{2} \\
& \geq\left\|d_{x}\right\|^{2}+\left\|d_{s}\right\|^{2} .
\end{aligned}
$$

The last inequality is due to the fact that

$$
\left\langle d_{x}, d_{s}\right\rangle=\frac{\langle\Delta x, \Delta s\rangle}{\mu}=\frac{\langle\Delta x, M \Delta x\rangle}{\mu} \geq 0,
$$


which follows from the monotonicity of the SCLCP (13). Equivalently, the same conclusion can be obtained by using the fact that $M$ and/or $\bar{M}$ are positive semidefinite. From (27), we see

$$
\left\|d_{x}+d_{s}\right\|=\|\nabla \Psi(v)\|=2 \delta(v) .
$$

By combining (48) and (50), we obtain

$$
4 \delta^{2}=\left\|d_{x}+d_{s}\right\|^{2} \geq\left\|d_{x}\right\|^{2}+\left\|d_{s}\right\|^{2} \geq\left\|d_{x}\right\|^{2} .
$$

Thus,

$$
\left\|d_{x}\right\|^{2} \leq 4 \delta^{2} \Rightarrow\left\|d_{x}\right\| \leq 2 \delta
$$

Similarly,

$$
\left\|d_{s}\right\|^{2} \leq 4 \delta^{2} \Rightarrow\left\|d_{s}\right\| \leq 2 \delta
$$

proving the lemma.

Using the above lemma the upper bound (46) for $f_{1}^{\prime \prime}(\alpha)$ can be significantly simplified.

Lemma 5.2 The following inequality holds:

$$
f_{1}^{\prime \prime}(\alpha) \leq 2 \delta^{2} \psi^{\prime \prime}\left(\lambda_{\min }(v)-2 \alpha \delta\right) .
$$

Proof By Lemmas 2.2 and 5.1 we have

$$
\eta_{i}=\lambda_{i}\left(v+\alpha d_{x}\right) \geq \lambda_{\min }\left(v+\alpha d_{x}\right) \geq \lambda_{\min }(v)-\left\|\alpha d_{x}\right\| \geq \lambda_{\min }(v)-2 \alpha \delta .
$$

Similarly, we obtain

$$
\gamma_{i}=\lambda_{i}\left(v+\alpha d_{S}\right) \geq \lambda_{\min }(v)-2 \alpha \delta
$$

Using the Peirce decompositions of $d_{x}$ (43) and $d_{s}$ (44) we may write

$$
\left\|d_{x}\right\|^{2}=\left\langle d_{x}, d_{x}\right\rangle=\sum_{i=1}^{r} d_{x_{i}}^{2}+\sum_{i<j} \operatorname{tr}\left(d_{s_{i j}}^{2}\right)
$$

and

$$
\left\|d_{s}\right\|^{2}=\left\langle d_{s}, d_{s}\right\rangle=\sum_{i=1}^{r} d_{s i}^{2}+\sum_{i<j} \operatorname{tr}\left(d_{x_{i j}}^{2}\right) .
$$

Since $\psi^{\prime \prime}(t)$ is monotonically decreasing and because of (46) it follows that

$$
f_{1}^{\prime \prime}(\alpha) \leq \frac{1}{2} \psi^{\prime \prime}\left(\lambda_{\min }(v)-2 \alpha \delta\right)\left(\sum_{i=1}^{r}\left(d_{x_{i}}^{2}+d_{s_{i}}^{2}\right)+\sum_{i<j}\left(\operatorname{tr}\left(d_{s i j}^{2}\right)+\operatorname{tr}\left(d_{x_{i j}}^{2}\right)\right)\right)
$$




$$
\begin{aligned}
& =\frac{1}{2} \psi^{\prime \prime}\left(\lambda_{\min }(v)-2 \alpha \delta\right)\left(\left\|d_{x}\right\|^{2}+\left\|d_{s}\right\|^{2}\right) \\
& \leq 2 \delta^{2} \psi^{\prime \prime}\left(\lambda_{\min }(v)-2 \alpha \delta\right) .
\end{aligned}
$$

The last inequality is due to (51) and the lemma is proved.

The subsequent lemmas lead to obtaining the default step-size. Given the proof of the above Lemma 5.2, their proofs are an easy modification of the similar lemmas stated in [1].

Lemma 5.3 (Lemma 5.5 in [1]) If the step size $\alpha$ satisfies the condition

$$
-\psi^{\prime \prime}\left(\lambda_{\min }(v)-2 \alpha \delta\right)+\psi^{\prime}\left(\lambda_{\min }(v)\right) \leq 2 \delta,
$$

then

$$
f_{1}^{\prime}(\alpha) \leq 0
$$

Lemma 5.4 (Lemma 5.6 in [1]) The largest possible value of the step size $\alpha$ satisfying (52) is given by

$$
\bar{\alpha}:=\frac{1}{2 \delta}[\rho(\delta)-\rho(2 \delta)]
$$

Lemma 5.5 (Lemma 5.7 in [1]) With $\rho$ and $\bar{\alpha}$ as defined above, we have

$$
\bar{\alpha} \geq \frac{1}{\psi^{\prime \prime}(\rho(2 \delta))} .
$$

Given the above lemma we use

$$
\tilde{\alpha}=\frac{1}{\psi^{\prime \prime}(\rho(2 \delta))}
$$

as the default step size in the analysis of the Generic Algorithm.

\subsection{Decrease of the Barrier Function During an Inner Iteration}

In this section we show that the default step size (55) yields sufficient decrease of the barrier function value during each inner iteration.

Lemma 5.6 (Lemma 5.10 in [1]) If the step size $\alpha$ is such that $\alpha \leq \bar{\alpha}$, where $\bar{\alpha}$ is defined by (53), then

$$
f(\alpha) \leq-\alpha \delta^{2}
$$

Using the default step size (55) we get the following immediate consequence of the above lemma. 
Theorem 5.2 If $\tilde{\alpha}$ is the default step size defined by (55), then

$$
f(\tilde{\alpha}) \leq-\frac{\delta^{2}}{\psi^{\prime \prime}(\rho(2 \delta))} .
$$

We need to express the bound on the decrease in terms of $\Psi$ instead of $\delta$. The basis to make the transition is the following theorem.

Theorem 5.3 (Theorem 5.9.12 in [11]) One has

$$
\delta(v) \geq \frac{1}{2} \psi^{\prime}(\varrho(\Psi(v))) .
$$

It first appeared in [28]. The version for LO over symmetric cones (SCLO) was first proved in [11] and it can be adopted for the SCLCP case without major changes. Furthermore, it worth mentioning that in its proof condition (32d) was used.

Next, we need the following simple but important lemma. In its proof condition (32c) was used.

Lemma 5.7 (Lemma 4.7 in [28]) The term

$$
-\frac{\delta^{2}}{\psi^{\prime \prime}(\rho(2 \delta))}
$$

in (56) is monotonically decreasing in $\delta$.

An immediate consequence is the following theorem.

Theorem 5.4 One has

$$
f(\tilde{\alpha}) \leq-\frac{\left(\psi^{\prime}(\varrho(\Psi(v)))\right)^{2}}{4 \psi^{\prime \prime}\left(\rho\left(\psi^{\prime}(\varrho(\Psi(v)))\right)\right)} .
$$

\section{Complexity of the Algorithm}

In this section, we find an upper bound on the total number of iterations of the Generic Algorithm based on the eligible kernel function and with the step size given by (55).

We first find an upper bound on the number of inner iterations needed to return to the situation where $\Psi(v) \leq \tau$ after a $\mu$-update. We denote the value of $\Psi(v)$ after the $\mu$-update as $\Psi_{0}$, and the subsequent values in the same outer iteration are denoted as $\Psi_{k}, k=1,2, \ldots, K$, where $K$ denotes the total number of inner iterations in the outer iteration. Recall that the decrease on each inner iteration is given by (57) in Theorem 5.4.

We make the following assumption: There exist positive constants $\beta$ and $\gamma \in] 0,1]$ such that

$$
f(\tilde{\alpha}) \leq-\frac{\left(\psi^{\prime}(\varrho(\Psi(v)))\right)^{2}}{4 \psi^{\prime \prime}\left(\rho\left(\psi^{\prime}(\varrho(\Psi(v)))\right)\right)} \geq \beta \Psi(v)^{1-\gamma} .
$$


Let us note that this assumption holds for $\gamma=1$ and

$$
\beta=\frac{\left(\psi^{\prime}(\varrho(\tau))\right)^{2}}{4 \psi^{\prime \prime}\left(\rho\left(\psi^{\prime}(\varrho(\tau))\right)\right)} \leq \frac{\left(\psi^{\prime}(\varrho(\Psi(v)))\right)^{2}}{4 \psi^{\prime \prime}\left(\rho\left(\psi^{\prime}(\varrho(\Psi(v)))\right)\right)}
$$

because $\Psi(v) \geq \tau>0$. However, this is the trivial case; our goal is to find $\gamma$ as small as possible for the reason that is explained in the Lemma 6.1 below. Furthermore, the appropriate values of $\beta$ and $\gamma$ will vary for each eligible kernel function and finding them may not always be straightforward.

The following lemma, adopted from [1], gives an upper bound on the number of inner iterations per one outer iteration.

Lemma 6.1 (Lemma 6.2 in [1]) If $K$ denotes the number of inner iterations per one outer iteration of the Generic Algorithm, then we have

$$
K \leq\left\lfloor\frac{\Psi_{0}^{\gamma}}{\beta \gamma}\right\rfloor
$$

The number of outer iterations coincides with the number of barrier parameter $\theta$ updates until we obtain $r \mu<\epsilon$. It is well known (Lemma П.17, p. 116 in [39]) that the number of outer iterations is bounded above by

$$
\frac{1}{\theta} \log \frac{r}{\epsilon} \text {. }
$$

Thus, an upper bound on the total number of iterations is the product of the upper bounds on the number of inner and the outer iterations.

Theorem 6.1 The total number of iterations $N$ in the Generic Algorithm is bounded from above by

$$
N \leq \frac{\Psi_{0}^{\gamma}}{\theta \beta \gamma} \log \frac{r}{\varepsilon}
$$

\section{Iteration Bounds}

\subsection{The Scheme}

As in $[1,11,28]$ the above analysis can be summarized in the following Scheme:

- Step 0: Specify a kernel function $\psi(t)$; an update parameter $\theta, 0<\theta<1$; a threshold parameter $\tau$; and an accuracy parameter $\epsilon$.

- Step 1: Solve the equation $-\frac{1}{2} \psi^{\prime}(t)=s$ to get $\rho(s)$, the inverse function of $\left.\left.-\frac{1}{2} \psi^{\prime}(t), t \in\right] 0,1\right]$. If the equation is hard to solve, derive a lower bound for $\rho(s)$.

- Step 2: Calculate the decrease of $\Psi(v)$ during an inner iteration in terms of $\delta$ for the default step size $\tilde{\alpha}$ from

$$
f(\tilde{\alpha}) \leq-\frac{\delta^{2}}{\left(\psi^{\prime \prime}(\rho(2 \delta))\right.} .
$$


- Step 3: Solve the equation $\psi(t)=s$ to get $\varrho(s)$, the inverse function of $\psi(t), t \geq 1$. If the equation is hard to solve, derive lower and upper bounds for $\varrho(s)$.

- Step 4: Derive a lower bound for $\delta$ in terms of $\Psi(v)$ by using

$$
\delta(v) \geq \frac{1}{2} \psi^{\prime}(\varrho(\Psi(v))) .
$$

- Step 5: Substitute the results of Steps 3 and 4 into Step 2 and find a valid inequality of the form

$$
f(\tilde{\alpha}) \leq-\beta \Psi(v)^{1-\gamma}
$$

for some positive constants $\beta$ and $\gamma$ with $\gamma \in] 0,1]$ as small as possible.

- Step 6: Calculate the upper bound of $\Psi_{0}$ from one of the inequalities

$$
\Psi_{0} \leq r \psi\left(\frac{\varrho\left(\frac{\tau}{r}\right)}{\sqrt{1-\theta}}\right) \leq \frac{r}{2} \psi^{\prime \prime}(1)\left(\frac{\varrho\left(\frac{\tau}{r}\right)}{\sqrt{1-\theta}}-1\right)^{2} .
$$

- Step 7: Calculate an upper bound for the total number of iterations by substituting results of Steps 5 and 6 into the expression

$$
\frac{\Psi_{0}^{\gamma}}{\theta \beta \gamma} \log \frac{r}{\epsilon} .
$$

- Step 8: Set $\tau=O(r)$ and $\theta=\Theta(1)$ to calculate a complexity bound for largeupdate methods, and set $\tau=O(1)$ and $\theta=\Theta\left(\frac{1}{\sqrt{r}}\right)$ to calculate a complexity bound for small-update methods.

The iteration bounds in Theorem 6.1 depend on the parameters $\beta$ and $\gamma$ and the upper bound on $\Psi_{0}$. Since these are different for different eligible kernel function, the iteration bounds will also vary. The iteration bounds for the specific eligible kernel function can be calculated using the Scheme. However, effort and skill are needed to find good bounds in certain steps of the Scheme, for example, in Steps 1, 3, and 4.

In next section, we use the Scheme to calculate iteration bounds of the Generic Algorithm for the kernel function $\psi_{10}$ in Table 1 . It is an easy modification of the similar analysis provided in [1] and is included here for illustration and completeness purposes.

\subsection{Iteration Bounds for $\psi_{10}(t)$}

As indicated, we consider the kernel function

$$
\psi_{10}(t)=\frac{t^{p+1}-1}{p+1}+\frac{t^{1-q}-1}{q-1}, p \in[0,1], q>1
$$

and provide detailed calculations of each step of the Scheme.

Step 1: To obtain the inverse function $t=\rho(s)$ of $-\frac{1}{2} \psi^{\prime}(t)=\frac{1}{2}\left(t^{p}-t^{-q}\right)$ for $t \in] 0,1]$ we need to solve $t$ from the equation

$$
-t^{p}+t^{-q}=2 s, \quad t \in(0,1] .
$$


Using that $t \leq 1$ we conclude that $t^{-q}=2 s+t^{p} \leq 2 s+1$, which implies

$$
t=\rho(s) \geq \frac{1}{(2 s+1)^{\frac{1}{q}}} .
$$

Step 2: Since $\psi^{\prime \prime}(t)=q t^{-q-1}$, it follows that

$$
\begin{aligned}
f(\tilde{\alpha}) & \leq-\frac{\delta^{2}}{\psi^{\prime \prime}(\rho(2 \delta))}=-\frac{\delta^{2}}{p(\rho(2 \delta))^{p-1}+\frac{1}{(\rho(2 \delta))^{q+1}}} \\
& \leq-\frac{1}{p(4 \delta+1)^{\frac{1-p}{q}}+q(4 \delta+1)^{\frac{q+1}{q}}} .
\end{aligned}
$$

Since $(4 \delta+1)^{1-p} \leq(4 \delta+1)^{q+1}$, for $p \in[0,1]$, and $q \geq 1$, it follows that

$$
f(\tilde{\alpha}) \leq-\frac{1}{(p+q)(4 \delta+1)^{\frac{q+1}{q}}} .
$$

Step 3: The inverse function of $s=\psi(t)$ for $t \in[1, \infty[$ is $t=\varrho(s)$. Thus, we have

$$
\frac{t^{1+p}-1}{1+p}=\frac{1-t^{1-q}}{q-1}+s,
$$

which leads to the following lower and upper bounds for $\varrho(s)=t$ :

$$
(1+(1+p) s)^{\frac{1}{1+p}} \leq \varrho(s)=t \leq\left((1+p) s+\frac{p+q}{q-1}\right)^{\frac{1}{1+p}} .
$$

Step 4: Now, using that $\delta(v) \geq \frac{1}{2} \psi^{\prime}(\varrho(\Psi(v)))$, and $\psi^{\prime}$ is monotonically increasing for $t \geq 1$, we may replace $\varrho(\Psi(v))$ by a smaller value. Thus, omitting the argument $v$, and assuming $\Psi \geq \tau \geq 1$, we obtain

$$
\begin{aligned}
\delta(v) & \geq \frac{1}{2}\left((1+(1+p) \Psi)^{\frac{p}{1+p}}-\frac{1}{(1+(1+p) \Psi)^{\frac{q}{1+p}}}\right) \\
& \geq \frac{1}{2}\left((1+(1+p) \Psi)^{\frac{p}{1+p}}-\frac{1}{(1+(1+p) \Psi)^{\frac{1}{1+p}}}\right),
\end{aligned}
$$

whence

$$
\delta(v) \geq \frac{(1+p) \Psi(v)}{2(1+(1+p) \Psi)^{\frac{1}{1+p}}} \geq \frac{\Psi(v)}{2(1+2 \Psi(v))^{\frac{1}{1+p}}} \geq \frac{1}{6} \Psi(v)^{\frac{p}{1+p}} .
$$

Step 5: Substituting (64) into (62) and noting that the right hand side expression in (62) is monotonically decreasing in $\delta$, we obtain

$$
f(\tilde{\alpha}) \leq-\frac{\Psi^{\frac{2 p}{1+p}}}{36(p+q)\left(\frac{2}{3} \Psi^{\frac{p}{1+p}}+1\right)^{\frac{q+1}{q}}} \leq-\frac{1}{100(p+q)} \Psi^{\frac{p(q-10)}{q 91+p)}} .
$$


Hence, we get $\beta=\frac{1}{100(p+q)}$ and $\gamma=\frac{q+p}{q(1+p)}$. Thus, the number $K$ of inner iterations per one outer iteration is bounded above by

$$
K \leq 100(1+p) q \Psi_{0}^{\frac{q+p}{q(1+p)}}
$$

Step 6: Now, we need to find an upper bound of $\Psi_{0}$. The fact that $\psi(t) \leq \frac{t^{1+p}}{1+p}$ for $t \geq 1$ leads to

$$
\Psi_{0} \leq n \psi\left(\frac{\varrho\left(\frac{\tau}{r}\right)}{\sqrt{1-\theta}}\right) \leq r \psi\left(\frac{\left(\frac{(1+p) \tau}{r}+\frac{q+p}{q-1}\right)^{\frac{1}{1+p}}}{\sqrt{1-\theta}}\right) \leq \frac{\left((1+p) \tau+\frac{q+p}{q-1} r\right)}{(p+1)(1-\theta)^{\frac{p+1}{2}}}
$$

Step 7: Hence, an upper bound for the total number of iterations is

$$
\frac{\Psi_{0}^{\gamma}}{\theta \bar{\beta} \gamma} \log \frac{r}{\epsilon} \leq \frac{100(1+p) q}{\theta(1-\theta)^{\frac{p+q}{2 q}}}\left(\frac{(1+p) \tau+\frac{q+p}{q-1} r}{1+p}\right)^{\frac{p+q}{q(1+p)}} \log \frac{r}{\epsilon} .
$$

Step 8: For large-update methods the right hand side expression becomes

$$
O\left(q r^{\frac{p+q}{q(1+p)}} \log \frac{r}{\varepsilon}\right)
$$

and for small-update methods

$$
O\left(q r^{\frac{p+q}{q(1+p)}+\frac{1}{2}} \log \frac{r}{\epsilon}\right)
$$

As we know, the iteration bound for small-update methods is usually better than the one for large-update methods. The reason why that is not the case here is because the upper bound for the inverse function $t=\varrho(s)$ of $s=\psi(t)$ is not sharp and can be improved as shown below. For that we need to go back to Step 3 .

Step 3: We have

$$
t \leq 1+\sqrt{t \psi(t)}
$$

Substituting $t \leq\left((1+p) \psi(t)+\frac{q+p}{q-1}\right)^{\frac{1}{1+p}}$ from (63) into the above inequality we obtain

$$
t=\varrho(s) \leq 1+\sqrt{s}\left((1+p) s+\frac{p+q}{q-1}\right)^{\frac{1}{2(1+p)}} .
$$

As a consequence Step 6 and the subsequent steps will change.

Step 6: We obtain the following upper bound of $\Psi_{0}$ :

$$
\Psi_{0} \leq \frac{r(p+q)}{2}\left(\frac{1+\sqrt{\frac{\tau}{r}}\left((1+p) \frac{\tau}{r}+\frac{p+q}{q-1}\right)^{\frac{1}{2(1+p)}}}{\sqrt{1-\theta}}-1\right)^{2} .
$$


By using the fact that

$$
1-\sqrt{1-\theta}=\frac{\theta}{1+\sqrt{1-\theta}} \leq \theta
$$

we get

$$
\begin{aligned}
\Psi_{0} & \leq \frac{r(p+q)}{2(1-\theta)}\left(\theta+\sqrt{\frac{\tau}{r}}\left((1+p) \frac{\tau}{r}+\frac{p+q}{q-1}\right)^{\frac{1}{2(1+p)}}\right)^{2} \\
& =\frac{p+q}{2(1-\theta)}\left(\theta \sqrt{r}+\tau\left((1+p) \frac{\tau}{r}+\frac{p+q}{q-1}\right)^{\frac{1}{2(1+p)}}\right)^{2} .
\end{aligned}
$$

Step 7: Thus, the total number of iterations is bound above by

$$
\frac{100(1+p) q}{\theta}\left(\left(\frac{p+q}{2(1-\theta)}\right)\left(\theta \sqrt{r}+\tau\left((1+p) \frac{\tau}{r}+\frac{p+q}{q-1}\right)^{\frac{1}{2(1+p)}}\right)^{2}\right)^{\frac{(p+q)}{q(1+p)}} \log \frac{r}{\epsilon}
$$

Since $\frac{p+q}{q(1+p)} \leq 1$ for all $p \in[0,1]$ and $q \geq 2$, the bound can be simplified to

$$
\frac{50 q(1+p)(p+q)}{\theta(1-\theta)}\left(\theta \sqrt{r}+\tau\left((1+p) \frac{\tau}{r}+\frac{p+q}{q-1}\right)^{\frac{1}{2(1+p)}}\right)^{2} \log \frac{r}{\epsilon} .
$$

Step 8: Now, for small-update methods and $p \in[0,1]$, the above expression becomes

$$
O\left(q^{2} \sqrt{r} \log \frac{r}{\varepsilon}\right)
$$

\subsection{Summary of Results}

In Table 2, we present the iteration bounds for all ten eligible functions listed in Table 1.

A few remarks are in order regarding the results in Table 2.

It turns out that the calculation of iteration bounds for monotone SCLCP is essentially the same as for the $P_{*}(\kappa)$-LCP (over the non-negative orthant) case presented in [1] except that $n$ is replaced by $r$ and the $\kappa$ term is dropped. Some of the results also match results for SCLO case in [11]. Although expected, these results were not obvious and, at certain steps of the analysis, they were not trivial and/or straightforward generalization of the LO case and the LCP over the non-negative orthant case.

For large-update methods the resulting iteration bounds differ in order of magnitude and are for some functions a significant improvement with respect to the iteration bound for the logarithmic barrier function. For $\psi_{2}$ and $\psi_{7}$ the bound is minimal if we choose $q=\frac{1}{2} \log r$. For $\psi_{10}$ the bound is minimal if we choose $p=1$ and $q=\frac{1}{2} \log r$. In both cases we obtain the best iteration bound known so far for these types of methods:

$$
O\left(\sqrt{r} \log r \log \frac{r}{\varepsilon}\right)
$$


Table 2 Complexity results for small- and large-update methods

\begin{tabular}{llll}
\hline$i$ & Kernel functions $\psi_{i}$ & Small-update methods & Large-update methods \\
\hline 1 & $\frac{t^{2}-1}{2}-\log t$ & $O\left(\sqrt{r} \log \frac{r}{\varepsilon}\right)$ & $O\left(r \log \frac{r}{\varepsilon}\right)$ \\
2 & $\frac{t^{2}-1}{2}+\frac{t^{1-q}-1}{q(q-1)}-\frac{q-1}{q}(t-1), q>1$ & $O\left(q \sqrt{r} \log \frac{r}{\varepsilon}\right)$ & $O\left(q r^{\frac{q+1}{2 q}} \log \frac{r}{\varepsilon}\right)$ \\
3 & $\frac{t^{2}-1}{2}+\frac{(e-1)^{2}}{e} \frac{1}{e^{t}-1}-\frac{e-1}{e}$ & $O\left(\sqrt{r} \log \frac{r}{\varepsilon}\right)$ & $O\left(r^{\frac{3}{4}} \log \frac{r}{\varepsilon}\right)$ \\
4 & $\frac{1}{2}\left(t-\frac{1}{t}\right)^{2}$ & $O\left(\sqrt{r} \log \frac{r}{\varepsilon}\right)$ & $O\left(r^{\frac{2}{3}} \log \frac{r}{\varepsilon}\right)$ \\
5 & $\frac{t^{2}-1}{2}+e^{\frac{1}{t}-1}-1$ & $O\left(\sqrt{r} \log \frac{r}{\varepsilon}\right)$ & $O\left(\sqrt{r} \log ^{2} r \log \frac{r}{\varepsilon}\right)$ \\
6 & $\frac{t^{2}-1}{2}-\int_{1}^{t} e^{\frac{1}{\xi}-1} d \xi$ & $O\left(\sqrt{r} \log \frac{r}{\varepsilon}\right)$ & $O\left(\sqrt{r} \log ^{2} r \log \frac{r}{\varepsilon}\right)$ \\
7 & $\frac{t^{2}-1}{2}+\frac{t^{1-q}-1}{q-1}, q>1$ & $O\left(q^{2} \sqrt{r} \log \frac{r}{\varepsilon}\right)$ & $O\left(q r^{\frac{q+1}{2 q}} \log \frac{r}{\varepsilon}\right)$ \\
8 & $t-1+\frac{t^{1-q}-1}{q-1}, q>1$ & $O\left(q^{2} \sqrt{r} \log \frac{r}{\varepsilon}\right)$ & $O\left(q r \log \frac{r}{\varepsilon}\right)$ \\
9 & $\frac{t^{1+p}-1}{1+p}-\log t, p \in[0,1]$ & $O\left(\sqrt{r} \log \frac{r}{\varepsilon}\right)$ & $O\left(r \log \frac{r}{\varepsilon}\right)$ \\
10 & $\frac{t^{p+1}-1}{p+1}+\frac{t^{1-q}-1}{q-1}, p \in[0,1], q>1$ & $O\left(q^{2} \sqrt{r} \log \frac{r}{\varepsilon}\right)$ & $O\left(q r^{\frac{p+q}{q(1+p)}} \log \frac{r}{\varepsilon}\right)$ \\
\hline
\end{tabular}

For small-update methods the resulting iteration bounds all have the same order of magnitude. Thus, the best iteration bound is the same as the iteration bound for the logarithmic kernel function:

$$
O\left(\sqrt{r} \log \frac{r}{\varepsilon}\right)
$$

which is the best known iteration bound for these types of methods.

\section{Concluding Remarks}

In this paper we provide a unified analysis of large- and small-update versions of the IPM for monotone SCLCP, described in Fig. 1, based on a large class of eligible kernel functions. The class of eligible kernel functions is important, because it is very general and includes the classical logarithmic kernel function, self-regular kernel functions, and a number of non-self-regular kernel functions as special cases.

The paper generalizes results obtained in [1], where we considered the same type of IPMs, however, only for LCPs over the non-negative orthant. The machinery of EJA and symmetric cones, as it relates to IPM and, in addition, to eligible kernel functions, had to be carefully used in order to obtain analogous results. In doing so, we relied on the results developed in [11], which is the only previous work that analyzes IPMs based on the eligible barrier (kernel) functions in the framework of EJA and symmetric cones. However, this work considers only LO over symmetric cones (SCLO). It turns out that the iterations bounds are the same as for the nonnegative orthant except that $n$ is replaced by $r$, the rank of the EJA. This is not either obvious or straightforward. For large-update methods the best iteration bound is $O\left(\sqrt{r}(\log r) \log \frac{r}{\varepsilon}\right)$ and for the small-update methods all iteration bounds have the same order of magnitude, namely, $O\left(\sqrt{r} \log \frac{r}{\varepsilon}\right)$, which is the best known iteration 
bound for these types of methods. Thus, the iteration bounds are as good as they can be in the current state-of-the-art.

It is worth mentioning that the results in [1] are obtained for $P_{*}(\kappa)$-LCPs over the non-negative orthant, while in this paper we consider monotone LCPs over symmetric cones. The generalization of the $P_{*}(\kappa)$ property to general symmetric cones is not straightforward, nor is it obvious. Development of such a property for symmetric cones is an interesting topic for further research.

The other issue that deserves comment is the assumption of strict feasibility of SCLCP. Strict feasibility, or the Interior Point Condition (IPC), as it is often called, can be assumed without loss of generality for LCPs over the non-negative orthant (Kojima et al. [17]). For the LO case, the same is true not only for the non-negative orthant but also in general; Luo, Sturm and Zhang in [40] discussed self-dual embedding for LO over symmetric cones. However, we are not aware of similar results for SCLCP. It certainly is a worthwhile research topic. Alternative direction for further research may be the development of infeasible kernel-based IPMs for SCLCP.

\section{References}

1. Lesaja, G., Roos, C.: Unified analysis of kernel-based interior-point methods for $P_{*}(\kappa)$-LCP. SIAM J. Optim. 20(6), 3014-3039 (2010)

2. Faraut, J., Koràny, A.: Analysis on Symmetric Cones. Oxford Mathematical Monographs. Clarendon/Oxford University Press, New York (1994)

3. Nesterov, Y., Nemirovski, A.: Interior-Point Polynomial Algorithms in Convex Programming. SIAM Studies in Applied Mathematics, vol. 13. SIAM, Philadelphia (1994)

4. Nesterov, Y., Todd, M.J.: Self-scaled barriers and interior-points methods for convex programming. Math. Oper. Res. 22(1), 1-42 (1997)

5. Nesterov, Y., Todd, M.J.: Primal-dual interior-point methods for self-scaled cones. SIAM J. Optim. 8(2), 324-364 (1998)

6. Güler, O.: Barrier functions in interior-point methods. Math. Oper. Res. 21(4), 860-885 (1996)

7. Faybusovich, L.: Linear systems in Jordan algebras and primal-dual interior-point algorithms. J. Comput. Appl. Math. 86(1), 149-175 (1997)

8. Faybusovich, L.: Euclidean Jordan algebras and interior-point algorithms. Positivity 1(4), 331-357 (1997)

9. Schmieta, S.H., Alizadeh, F.: Associative and Jordan algebras, and polynomial time interior-point algorithms for symmetric cones. Math. Oper. Res. 26(3), 543-564 (2001)

10. Schmieta, S.H., Alizadeh, F.: Extension of primal-dual interior-point algorithms to symmetric cones. Math. Program. 96(3), 409-438 (2003)

11. Vieira, M.: Jordan algebraic approach to symmetric optimization. Ph.D. Thesis, TU Delft (2007)

12. Gu, G.: Interior-point methods for symmetric optimization. Ph.D. Thesis, TU Delft (2009)

13. Anitescu, M., Lesaja, G., Potra, F.A.: An infeasible interior-point predictor-corrector algorithm for the $P_{*}$-geometric LCP. Appl. Math. Optim. 36, 203-228 (1997)

14. Anitescu, M., Lesaja, G., Potra, F.A.: Equivalence between different formulations of the linear complementarity problem. Optim. Methods Softw. 7, 265-290 (1997)

15. Bai, Y.Q., Lesaja, G., Roos, C.: A new class of polynomial interior-point algorithms for linear complementarity problems. Pac. J. Optim. 4(1), 19-41 (2008)

16. Liu, X., Potra, F.A.: Corrector-predictor methods for sufficient linear complementarity problems in a wide neighborhood of the central path. SIAM J. Optim. 17(3), 871-890 (2006)

17. Kojima, M., Megiddo, N., Noma, T., Yoshise, A.: A Unified Approach to Interior Point Algorithms for Linear Complementarity problems. Lecture Notes in Computer Science, vol. 538. Springer, New York (1991)

18. Miao, J.: A quadratically convergent $O((1+\kappa) \sqrt{n} L)$-iteration algorithm for the $P_{*}(\kappa)$-matrix linear complementarity problem. Math. Program. 69, 355-368 (1995) 
19. Potra, F.A.: A superlinearly convergent predictor-corrector method for degenerate LCP in a wide neighborhood of the central path with $O(\sqrt{n} L)$-iteration complexity. Math. Program., Ser. A 100(2), 317-337 (2004)

20. Wright, S.J., Zhang, Y.: A superquadratic infeasible-interior-point method for linear complementarity problems. Math. Program. 73, 269-289 (1996)

21. Ye, Y.: On homogeneous and self-dual algorithms for LCP. Math. Program. 76, 211-221 (1997)

22. Kojima, M., Shindoh, S., Hara, S.: Interior-point methods for the monotone semidefinite linear complementarity problems in symmetric matrices. SIAM J. Optim. 7(1), 86-125 (1997)

23. Monteiro, R.D.C., Tsuchiya, T.: Polynomiality of primal-dual algorithms for semidefinite linear complementarity problem based on the Kojima-Shindoh-Hara family of directions. Math. Program. 84, 39-53 (1999)

24. Tseng, P.: Search directions and convergence analysis of some infeasible path-following methods for monotone semidefinite LCP. Optim. Methods Softw. 9(4), 245-268 (1998)

25. Rangarajan, B.K.: Polynomial convergence of infeasible-interior-point methods over symmetric cones. SIAM J. Optim. 16(4), 1211-1229 (2006)

26. Yoshise, A.: Interior-point trajectories and a homogeneous model for nonlinear complementarity problems over symmetric cones. SIAM J. Optim. 17, 1129-1153 (2006)

27. Yoshise, A.: Homogeneous algorithms for monotone complementarity problems over symmetric cones. Pac. J. Optim. 5, 313-337 (2009)

28. Bai, Y.Q., El Ghami, M., Roos, C.: A comparative study of kernel functions for primal-dual interiorpoint algorithms in linear optimization. SIAM J. Optim. 15(1), 101-128 (2004)

29. Baes, M.: Spectral functions and smoothing techniques on Jordan algebras. Ph.D. Thesis, Catholic University of Louvain (2006)

30. Chung, S.J.: A note on the complexity of LCP. The LCP is strongly NP-complete. Technical Report No. 792, Department of Industrial and Operations Engineering, The University of Michigan (1979)

31. Alizadeh, F., Haeberley, J.-P.A., Overton, M.L.: Primal-dual interior-point methods for semidefinite programming: convergence rates, stability and numerical results. SIAM J. Optim. 8(3), 746-768 (1998)

32. Helmberg, C., Rendl, F., Vanderbei, R.J., Wolkowiwicz, H.: An interior-point method for semidefinite programming. SIAM J. Optim. 6(2), 342-361 (1996)

33. Monteiro, R.D.C., Zhang, Y.: A unified analysis for a class of long-step primal-dual path-following interior-point algorithms for semidefinite programming. Math. Program. 81(3), 281-299 (1998)

34. Muramatsu, M.: On a commutative class of search directions for linear programming over symmetric cones. J. Optim. Theory Appl. 112(3), 595-625 (2002)

35. Lim, Y.: Geometric means on symmetric cones. Arch. Math. (Basel) 75(1), 39-45 (2000)

36. Peng, J., Roos, C., Terlaky, T.: Self-Regularity: A New Paradigm for Primal-Dual Interior-Point Algorithms. Princeton University Press, Princeton (2002)

37. Bai, Y.Q., Roos, C.: A polynomial-time algorithm for linear optimization based on a new simple kernel function. Optim. Methods Softw. 18(6), 631-646 (2003)

38. Bai, Y.Q., Lesaja, G., Roos, C., Wang, G.Q., El Ghami, M.: A class of large- and small-update primaldual interior-point algorithms for linear optimization. J. Optim. Theory Appl. 138(3), 341-359 (2008)

39. Roos, C., Terlaky, T., Vial, J.Ph.: Theory and Algorithms for Linear Optimization. An Interior-Point Approach. Springer Science. Springer, New York (2005)

40. Luo, Z.Q., Sturm, J.F., Zhang, Y.: Conic convex programming and self-dual embedding. Optim. Methods Softw. 14, 169-218 (2000) 